(18 patients). The average follow up was 4 months. Most of the patients were treated with capecitabine-oxaliplatin, followed by those treated with capecitabine monotherapy and other minority schemes (cyclophosphamide or bevacizumab). The median starting dose of capecitabine was $3300 \mathrm{mg}$.

$32 \%$ of patients required a dose adjustment (delay and/or dose reduction) during the follow-up period. The treatment of $26 \%$ of patients was delayed by an average of 16 days ( 2 of the patients had to delay 2 cycles). The initial dose was reduced in $24 \%$ of patients (twice in three of the patients).

Toxicity in any grade was reported by $30 \%$ of the patients. Severe toxicities (grade 3 of CTCAE) were sickness and neutropenia. Most frequent toxicities were gastrointestinal side effects ( 6 patients) and grade 2 hand-foot syndrome (4 patients), followed by mucositis, skin side effects, hyperbilirubinaemia and thrombopenia.

Toxicity and dose adjustment were not statistically related to the treatment regimen, ECOG, gender or age.

Conclusions The toxicity profile was consistent with the trials. $81 \%$ of patients who had a dose adjustment didn't need a further dose reduction.

No conflict of interest.

\section{CPC-072 INCLUSION OF PHARMACOGENETICS STUDIES, PATIENT- REPORTED OUTCOMES AND COST MEASURES IN CLINICAL TRIALS; VARIABLES ADDED IN RECENT YEARS}

doi:10.1136/ejhpharm-2013-000276.529

M Bovaira-García, P Mira-García, A Llopis-Anduix, A Biel-Sanchís, A Mondaray-Tormo, E Soler-Company, R Olivares-Pallerols, JP Navarro-Ferrando, C Sangrador-Pelluz. Hospital Arnau de Vilanova, Pharmacy, Valencia, Spain

Background Pharmacogenetic studies analyse the variability of drug response; patient-reported outcome (PRO) measures complement traditional measures. Pharmacoeconomic studies tell us the efficiency of different therapeutic alternatives.

Purpose To evaluate the use of PRO measures, including healthrelated quality of life questionnaires (HROoLO), and the frequency of inclusion of pharmacogenetics studies and economic variables in the design of clinical trials (CT) and observational studies (ST). For CT, the quality of the study design was also measured.

Materials and Methods Observational study of CT and ST approved by a Clinical Research Ethics Committee (active between Jan/2008-May/2012). Information recorded: medical specialty, pathology, methodological quality (Jadad scale: 0-5), inclusion of PRO, HRQoLQ, pharmacogenetics studies (collection or not of human biological samples) and economic variables (use of healthcare resources and/or indirect costs defined as the number of days lost due to sick leave of patients and caregivers). The information was systematically collected by 2 reviewers and checked by a third if discrepancies arose

Results Ninety-four protocols (79CTs, 15ST) were analysed; 51 included PRO measures (54.3\%), 44 CT (38 had HRQoLO) and 7 ST (6 had HRQoLQ). Analysis by area showed PRO measures were most commonly studied in: endocrinology, neurology, digestive diseases and cardiology. The average quality score was 3.04. 31 studies incorporated pharmacogenetics studies, which were less frequent before 2010 than after (45.3\% versus $65.4 \%$ ). In 50\% of the pharmacogenetics studies the storage of collected human biological material in biobanks was planned with the objective of conducting studies about drugs responses according to the genetic endowment. Twenty (25\%) CTs and two (18.2\%) STs included economic variables.

Conclusions The evaluation of economic variables in CTs and STs was low. More than half of the protocols included PRO measures, reflecting the importance of these parameters. Increasing knowledge of pharmacogenetics has resulted in a higher inclusion of these studies in more recent CTs. The average quality for the CT exceeded the value 3 , indicating that studies analysed were of reasonable quality.
No conflict of interest.

\section{CPC-073 INFLUENCE OF FIRST-LINE EGFR THERAPY ON SURVIVAL AND MORTALITY RATES IN NON-SMALL CELL LUNG CANCER}

doi:10.1136/ejhpharm-2013-000276.530

${ }^{1} \mathrm{C}$ Pérez Ramírez, ${ }^{1} \mathrm{M}$ Cañadas Garre, ${ }^{2} \mathrm{R}$ López Castro, ${ }^{2} \mathrm{~A}$ Concha López, ${ }^{1} \mathrm{MA}$ Calleja Hernández. 'Virgen de las Nieves University Hospital, Pharmacy Service. Pharmacogenomics Unit, Granada, Spain; '2Virgen de las Nieves University Hospital, Pathology Department, Granada, Spain

Background The efficacy of chemotherapy has reached a plateau for advanced non-small cell lung cancer (NSCLC). Increasing evidence has demonstrated that patients with sensitising mutations in the epidermal growth factor receptor (EGFR) are associated with sensitivity to reversible EGFR tyrosine kinase inhibitors (TKIs). Numerous studies have demonstrated improvement of progressionfree survival compared to conventional chemotherapy as first-line treatment for advanced NSCLC with EGFR mutations.

Purpose To evaluate mortality and overall survival (OS) in NSCLC patients treated with EGFR-TKIs or chemotherapy according to their EGFR status.

Materials and Methods Retrospective study. Sixty-one patients diagnosed with NSCLC and available EGFR status during 20082012 were included. Socio-demographic, clinical and pharmacological characteristics of patients were collected. Comparison of medians by Mann-Whitney-Wilcoxon Test for numerical variables and Chi-Square Test for categorical variables was performed.

Results Mean age was $62 \pm 12$ years; $52.5 \%$ (32/61) male; $70.5 \%$ (43/61) smokers/ex-smokers; 60.7\% (37/61) stage IV; 42.6\% (23/54) mutant EGFR. Minimum follow-up of 6 months was accomplished in 54 patients.

An EGFR-TKI was prescribed as first-line treatment in $65.2 \%$ $(15 / 23)$ EGFR-positive patients, $80.0 \%(12 / 15)$ stage IV, with an OS of $12.40[11.30-23.33]$ months and $53.3 \%(8 / 15)$ deaths. Two patients required second-line chemotherapy $(2 / 15 ; 13.3 \%)$.

Chemotherapy as first-line treatment was prescribed in $75 \%$ patients (46/61), 17\% EGFR-positive (8/46), 50.0\% (4/8) stage IV, with $29 \%(2 / 7)$ deaths. EGFR-TKIs were used as second-line treatment in $87.5 \%(7 / 8)$ patients and third-line in $12.5 \%(1 / 8)$. OS was 17.97[8.83-60.84] months.

EGFR was native in $67.4 \%$ (31/46) patients, $58.1 \%$ (18/31) stage IV, and $61.3 \%(19 / 31)$ deaths. EGFR-TKIs as second-line treatment were prescribed in $61.3 \%$. (19/31) patients, third-line in $35.5 \%$ $(11 / 31)$ and fourth-line in $3.2 \%(1 / 31)$.

Seven patients had unknown EGFR status (7/61; 11.5\%), 57.1\% (4/7) stage IV, and $42.8 \%$ (3/7) deaths. EGFR-TKI as second-line treatment was prescribed in $85.7 \%(6 / 7)$ patients and fourth-line treatment in $14.3 \%(1 / 7)$.

OS and mortality were not statistically different between EGFRpositive patients treated with EGFR-TKIs/chemotherapy as firstline treatment $(P=0.836 ; p=0.105)$. Mortality was not associated with stage or EGFR status ( $\mathrm{P}=0.086 ; \mathrm{p}=1.000)$.

Conclusions Mortality and OS are not associated with EGFR status or stage in this NSCLC population. EGFR-positive patients present similar OS and mortality rates regardless of first-line treatment.

No conflict of interest.

\section{CPC-074 INTENSIVE MONITORING OF ADVERSE REACTIONS IN ONCOHEMATOLOGY: PROJECT FARMAREL}

doi:10.1136/ejhpharm-2013-000276.53

A Ragazzi, 'L Casorati, 'VM Conte, 'M Savoldelli, 'MG Ottoboni, 'D Ferla, 'T Testa, ${ }^{2 M C}$ Pasquini, ${ }^{2} \mathrm{~A}$ Inzoli. "Ospedale Maggiore" di Crema, Pharmacy, Crema, Italy, 2"Ospedale Maggiore" di Crema, Oncology, Crema, Italy 